\title{
Social Media for Crisis Communication in the Coronavirus (COVID-19) Outbreak: A Study of NCDC
}

\author{
Beryl EHONDOR, PhDc
}

School of Media \& Communication

Pan-Atlantic University, Lagos

E-mail: beryl.ehondor@pau.edu.ng

\section{Christiana UNAKALAMBA}

School of Media \& Communication

Pan-Atlantic University, Lagos

E-mail: christiana.unakalamba@pau.edu.ng

\begin{abstract}
Several scholars have studied social media use for crisis communication. However, few studies have been carried out to investigate social media deployment during a public health crisis, such as the Coronavirus outbreak. This study accessed public awareness and engagement of Nigeria Centre for Disease Control (NCDC)'s social media use for crisis communications during the Coronavirus outbreak in Nigeria. It also assessed the influence of communication towards public positive behavioural adjustments. The study data was gathered via a survey of 400 citizens and Facebook users and analysed using excel and SPSS. The study found public awareness of the NCDC social media communications during the outbreak; there was also a public behavioural adjustment to NCDC's recommended preventive behaviours. However, there was a low engagement in NCDC's crisis communication via Facebook. The study reveals a high level of public uncertainty about NCDC Facebook communications and a change in public
\end{abstract}


opinion about the Coronavirus. This study concludes that this situation could result from competing information about the Coronavirus on social media during the COVID outbreak and recommended further studies in this area. Findings are helpful for health communication policy reviews, strategic crisis communication assessments, appraisal of similar agencies, and further research.

Keywords: Crisis-communication; Social-media; Coronavirus-outbreak; Nigeria Centre for Disease Control (NCDC); Infodemics.

\section{Introduction}

The declaration of COVID-19 as a pandemic in March 2020 by the World Health Organisation (henceforth WHO) has progressed into a global crisis (WHO, 2020; Ghebreyesus, 2020). Nations have experienced tragic health hazards, economic uncertainties, and social disruptions that have resulted in a high cost to international and national economies. In a few weeks of the Coronavirus outbreak, the authors observed that the world was pushed to the height of a more severe recession than the 2008 financial crisis due to the lockdown measures to manage the Coronavirus spread. Major industries, mainly airlines, hotels, tourism, hospitality, and other entertainment-related sectors, are almost bankrupt. Also, this has led to widespread business failures and joblessness.

Nigeria entered the COVID-19 statistics after the first recorded case of the Coronavirus in Abeokuta, South of the nation. It involved an Italian who worked in $\mathrm{Ni}$ geria and returned from Milan, Italy, to Lagos, Nigeria, on 25 February 2020 (BBCBritish Broadcasting Corporation, 2020). Since then, there has been a rapid spread around the country, and a series of sharp drastic changes have begun to develop in economic, social and industrial activities. Such occurrences have become a turning point for worse, creating heightened uncertainties such as; the lockdown measures, curfews limiting movements and interactions, the country's looming economic crisis as oil price collapse, deaths, etc.

The Coronavirus pandemic was unexpected, unplanned, and threatens the stability of businesses and organisations. It has caused drastic changes within many organisations, stretching their resources, halted operations, caused illness and deaths. The outbreak turned into a pandemic, causing a social, economic, and global crisis. Consequently, information, timely updates, and communication about the Coronavirus are critical aspects of managing the situation. There is usually a barrage of information about the disease in a crisis, thus generating sometimes untraceable and unreliable messages (Tran, Valecha, \& Rad, 2020). Since the Coronavirus pandemic's eruption in December 2019 in Wuhan, China, myriads of confirmed and unconfirmed online messages have gone viral on the disease's meaning (Chen, 
Luo, Hu, Zhao, \& Zhang, 2021), origin, management, and implications (Banerjee \& Meena, 2021).

This constitutes a challenge for the public in sourcing and accepting social media information regarding the Coronavirus. Since the World Health Organisation declared the Coronavirus disease a global pandemic on 11 March 2020, nations, societies and communities are still devising approaches to survive by establishing organisations and agencies responsible for information management and communication within the outbreak beyond. Nigeria already had the Nigeria Centre for Disease Control. NCDC played a significant role in the battle against the Ebola pandemic in the country six years ago. NCDC was set up with one of its core functions: collating, synthesising, and disseminating public health research to inform policy. In achieving this mandate, the NCDC deploys several avenues such as the mainstream media and the online platform inclusive of social media (Shahaduzzaman et al., 2020; NCDC, 2020)

Social media played a crucial role in determining public response to communications, especially regarding the COVID pandemic. This is due to the interactive nature of social media as a platform for general communications and interactions. It further portends a vast opportunity in its use to reach all classes of individuals and groups. Social media platforms such as websites for social networking and microblogging such as Facebook, Instagram, Twitter, and others provide platforms through which users create online communities to share information, ideas, personal messages, and other content (such as videos).

Therefore, the influence of social media messages and information regarding the Coronavirus, if deployed effectively, could affect public behaviour towards the disease. At the same time, the inefficient use of these platforms hinders its potentials. It may not effectively compete nor counter the deluge of unreliable data published on social media platforms, which are often inaccurate and untraceable.

Intentionally or otherwise, social media presented the potential for significant dissemination and misinformation about COVID-19 source, statistics, transmission methods, prevention, and medications for the virus. Scholars say this has led to a situation which is known as "Misinfodemics". Misinfodemics, according to (Sahni \& Sharma 2020), is concerned with misinforming and creating panic. Some persons knowledgeable or not leveraged social media to magnify their opinions. Such opinions may travel wide and are assessed by a great many individuals. Therefore, given the critical role social media play in the growing health crisis caused by the COVID pandemic, this study focuses on social media deployment for crisis communication during the Coronavirus outbreak in Nigeria.

Since the WHO declaration of the Coronavirus outbreak as a global pandemic, nations have devised avenues to manage and thrive despite the economic impact, confusion, uncertainties, health risk, and exposures due to the Coronavirus outbreak. No matter how brief, situations like this create a crisis for nations, organisations, 
and individuals. In such a desperate time, communications directly impact the public and invariably determine whether the public panic escalates or relaxes. The news of the first Coronavirus case and subsequent updates on increased spread caused the government to rely on one of its agencies to spearhead communications involving updates and enlightenment about the Coronavirus. The NCDC has been at the forefront of projecting, publishing, and disseminating data/updates about the Coronavirus. In addition, the NCDC helps the government achieve its aim of managing the crisis during the Coronavirus outbreak through social media contents and publications (Nigeria Centre for Disease Control, 2020).

Even though past studies have examined media use in crisis communication, scholars established that organisations could achieve more by using social media. Dunbar et al. (2015) point out that many organisations have gained mileage by using social media platforms to communicate in a faster way with their publics during emergencies as well as responding to queries and questions. Therefore, this study will examine the engagement of the NCDC social media use during the Coronavirus outbreak in Nigeria and public response to it. Lots of prior research focused on various approaches to crisis communication and public response. However, only a few academic materials tackle a public health crisis such as the Coronavirus, especially in the Nigerian context, thus creating a knowledge gap that this research will attempt to fill.

The research questions of the study are the following:

RQ 1: What is the level of public awareness on NCDC COVID-19 crisis communication via Facebook during the COVID-19 lockdown?

RQ 2: What is the level of engagement between NCDC COVID-19 crisis communication via Facebook and Nigerians?

RQ 3: What is the influence of NCDC crisis communication via Facebook on Nigerians' (reported) positive health behavior?

This research was restricted within social media scope (focusing on Facebook social media handle) for communication during the Coronavirus outbreak. The choice of Facebook was based on preliminary research, which shows that NCDC most popular social media handle used for communicating during the lockdown was via Facebook. Also, Facebook had and still has the most significant subscribers among all the NCDC's social media platforms during the lockdown. The time scope is explicitly focused during the lockdown period from the first date of government announcement of lockdown measure-30 March 2020 to 1 June 2020, which constitutes the first lockdown phase characterised by a complete lockdown. Thus, the study focuses on the social media communication dimension and approach during an impending health crisis.

The study fills the gap in the academic literature on Social media use for Health Crisis Communication in Nigeria. This is because, as at the time of this study, out 
of the few studies that have been carried out on Public Health Crisis communication, especially within the Nigerian context and not many studies have delved into the social media use for crisis communication by a governmental agency during the COVID pandemic.

Hence, this paper benefits the government, helps policymakers and public health agencies understand, determine, and know how to use social media to communicate more effectively during a health crisis. This will equally help manage the effects of the Coronavirus on the economy. This study will make it easier to determine the relevance and engagement of funds used for social media communications. This work will also provide a future reference in measuring the NCDC social media communications efficiency during the Coronavirus outbreak in Nigeria. Furthermore, the impact of public communications (most especially social media communications) on the public inadvertently impacts their economic choices, behaviour, and decisions. This study provides timely data in determining a strategic position in crisis communication within public health issues within the Nigerian context.

Finally, with the development of the Coronavirus in Africa, the importance of strategic public health crisis communication cannot be overemphasised. This study becomes even more significant as government, organisations, scholars, PR experts continue to devise ways to communicate information, data, developments, and updates about the Coronavirus. In addition, the study provides a framework for them to determine how to use social media to communicate to the public during a public health crisis, especially during the COVID-19 eras. Thus, this study will be significant and relevant to the health sector in Nigeria, SDG's, government, and social workers. It will help them gauge how to deploy social media platforms in addressing public health crisis issues in Nigeria.

\section{Theory of Protective Motivation Theory (PMT)}

The Protection Motivation Theory (PMT), by Rogers in 1975, is one of the scholars' theories to explain risk perception (Rogers, 1975; Rogers \& Prentice-Dunn, 1997). It is somewhat similar to the Health belief theory as it connects fear, risk and behaviour change. This theory showcases a person's likelihood to protect himself due to the anticipation of adverse outcomes. According to Shaw (2012), The Protection motivation theory modifies the health belief theory founded by R.W. Rogers in 1975 (Wong et al., 2016). However, the theory was initially based on Richard Lazarus work on how people act and cope during stressful situations (Zimmer-Gembeck \& Skinner, 2016). Richard Lazarus stated that people are different in their sensitivity and vulnerability to specific events and respond differently. People consider the severity of an event, their vulnerability, and the benefits of undertaking protective actions before deciding to expose themselves to risk. Generally, PMT assumes that there is a connection between public risk perception and 
protective action (Westcott et al., 2017; Bubeck et al., 2018). Shereen et al. (2013) found that enhancing risk perception and perceived severity significantly affects intentional and behavioral change toward safety.

According to the protection motivation theory, public awareness of risk and personal protective equipment is linked. For instance, public awareness of deaths and the number of COVID-19 infected cases tend to induce public concern and motivation for self-protection and the use of protective tools to prevent risk. Shaw (2012) noted that PMT is deployed to emphasise safety campaigns, and messages resulting from PMT were based on raising awareness of risk exposure consequences and that being motivated to protect oneself requires adequate risk perception. In applying PMT theory to the study on social media use for crisis communication during the Coronavirus, public motivation to protect themselves and indulge in preventive behaviours is dependent on perceived risk to the virus based on information gathered via the media or agency communication on COVID19.

Therefore, public participation in risk preventative behaviours is dependent on their motivation to protect themselves from threats such as natural disasters. Janmaimool (2017) states that; this decision is made based on two results which are: (1) threat appraisal and (2) coping appraisal. Threat appraisal involves a process people use to appraise the level of threat or risk they are exposed to, which involves assessing vulnerability. Thus, information and knowledge gathered on the Coronavirus can help an individual determine their level of vulnerability.

Based on this theory's principles, how these messages are crafted can also induce individuals perceived severity and perceived vulnerability. The second, coping appraisal, states that the appraisal of an individual's capability to carry out risk preventative behaviours influences protection motivation. Therefore, how individuals perceive their ability to perform preventive behaviours will influence response to recommended risk preventative behaviours. This implies that how individuals perceive their capacity to carry out NCDC recommended COVID-19 preventive behaviour will largely determine if they would adhere. Hence, public perception of the risk of infection of the COVID-19 and ability to engage in preventive behaviour is inadvertently linked to the nature of the information they access about the Coronavirus.

\section{Methodology}

Based on empirical findings, the quantitative approach to data collation adopted for this research is the survey method. The study is descriptive, and the survey method allows for generalisation, collation, and data collection from a broader respondent base. This method also eliminates the interviewer's bias, as it offers a higher degree of confidentiality. The population under study is made up of Nigerians who are social media users. Based on current statistics on social media 
use in Nigeria, 85.49 million Nigerians use the internet and 27 million of these are social media users. According to Datareportal (2020), the number of social media users in Nigeria increased by 3.4 million (+14\%) between April 2019 to January 2020. Besides WhatsApp, Facebook is the following social media with the secondhighest number of users, with $87 \%$ of Nigeria's total social media users.

Since part of the study focuses on social media use, an essential requirement for choosing the sample is that respondents should be active on social media. This means they have access to social media to qualify to be tested on messages about the COVID-19 deployed by the NCDC deploys on such platforms. Therefore, the sample population was chosen using the random sampling method. This sampling method involves selecting a sample population based on several factors, including their availability and willingness to participate in the research and access social media platforms.

The sample size of 400 was derived using the Yamane formula of sample calculation. The instruments used to gather the data for this research is a questionnaire. The questionnaire was sent to 400 respondents online via a Google form link sent to respondents. The questionnaire consisted of questions testing respondents' exposure to NCDC social media messages on Coronavirus, the engagement of these social media messages about the Coronavirus, and how NCDC messages on COVID-19 have influenced the positive health behaviour of the public.

Microsoft Excel and the statistical package for the social sciences (SPSS) were used to compute and analyse the research data.

\section{Instrument description}

The questionnaire was segmented into sections $A-D$ to address the research questions; Section A covered demographics, while sections B, C and D covered research questions one to three, respectively. Sections B and C provided data on the awareness of NCDC's social media messages on COVID19 during the lockdown, the engagement of NCDC's social media messages during the lockdown. It is noteworthy that Section C further narrowed down testing of NCDC social media to Facebook. Since Facebook has the most significant social media usage in Nigeria and has many NCDC social media subscribers/following. Finally, Section D focused on determining if the NCDC social media use for COVID-19 communication influenced positive health behaviour during the lockdown.

\section{Sample}

All findings were collated and analysed using Google sheet and excel. The data is based on a sample population of 400 . The total number of questionnaires retrieved was 400. The discussion of results helps to answer the research questions.

The findings indicate more females than males on NCDC social media. Also, $43.8 \%$ of respondents were between the ages of $26-35$, while $25 \%$ were between 
ages 36-45year, and $21.8 \%$ were between ages 45 and above. While the last are those between the ages 18-25years, who constitute $9.5 \%$ of the total population. Furthermore, $93.7 \%$ are active on social media. Even though the remaining $6.3 \%$ are social media users but not active. The most frequented social media platform of respondents is Facebook.

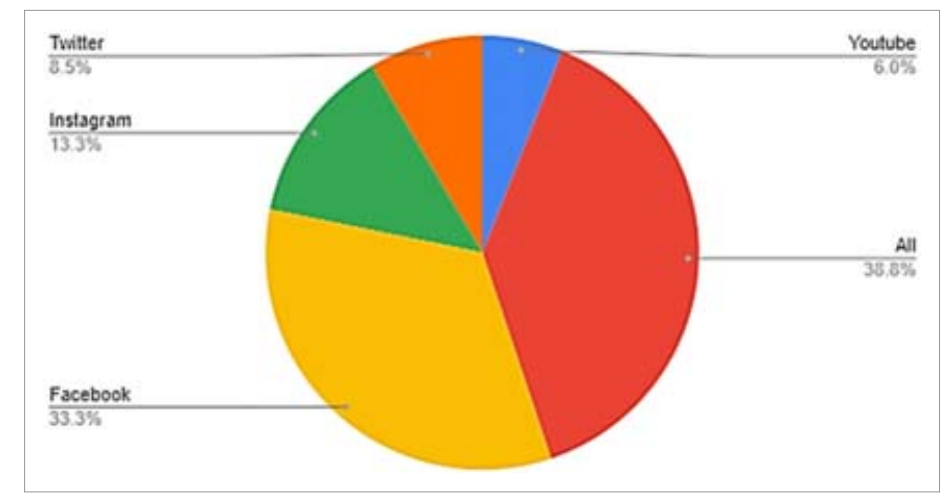

Figure 1. Popular social media platforms respondents are active on

Source: Fieldwork, 2020

\section{Results}

This research tested the effectiveness of social media for crisis communication in COVID-19, within a local context. The study further examined how NCDC messaging on COVID-19 influenced positive health behaviour or otherwise.

This section presents the data gathered from the survey, analysis, interpretation, and findings in line with the research questions posed at the beginning of this study. In collecting the data, the authors deployed a random sampling of social media users. This was administered via social media platforms. This was necessary due to the Coronavirus pandemic social restrictions. Moreover, the research focus is on social media use.

\section{Awareness of NCDC and Messaging on COVID-19} via social media during the lockdown

The question of awareness of existence and function sought to gauge the level of public awareness of the NCDC. Testing public awareness of NCDC guided the authors in determining NCDC crisis communication's popularity and public awareness. $48 \%$ of respondents indicated that they are highly aware of NCDC, $29.75 \%$ said they are very aware, while $14.25 \%$ stated they are moderately aware of NCDC.

However, $5.75 \%$ and $2 \%$ of the respondents indicated that they are slightly aware and unaware of the NCDC. This suggests that to a large extent, NCDC is known 


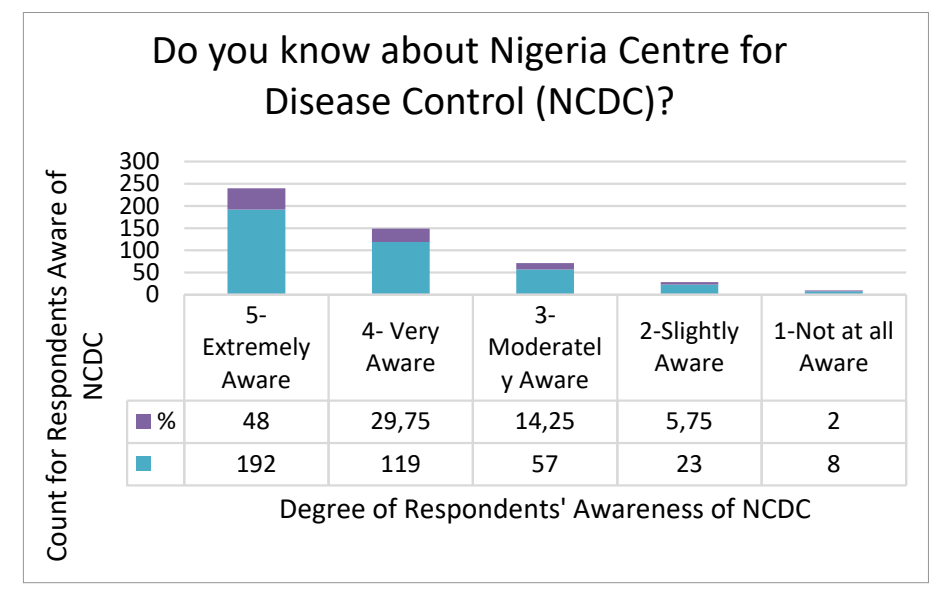

Figure 2. Public knowledge of the Nigeria Centre for Disease Control (NCDC)

Source: Fieldwork, 2020

in Nigeria as an overwhelming number of the sample population (92\%) are aware while an insignificant part of the population (8\%) are not so aware of NCDC. This indicates that Nigeria's NCDC activities may be very low context (focused mainly on the words being said/ explicit communication). The $25.93 \%$ of respondents who indicated $\mathrm{n} / \mathrm{a}$ is representative of those who may never have had any cross-cultural communication misunderstandings.

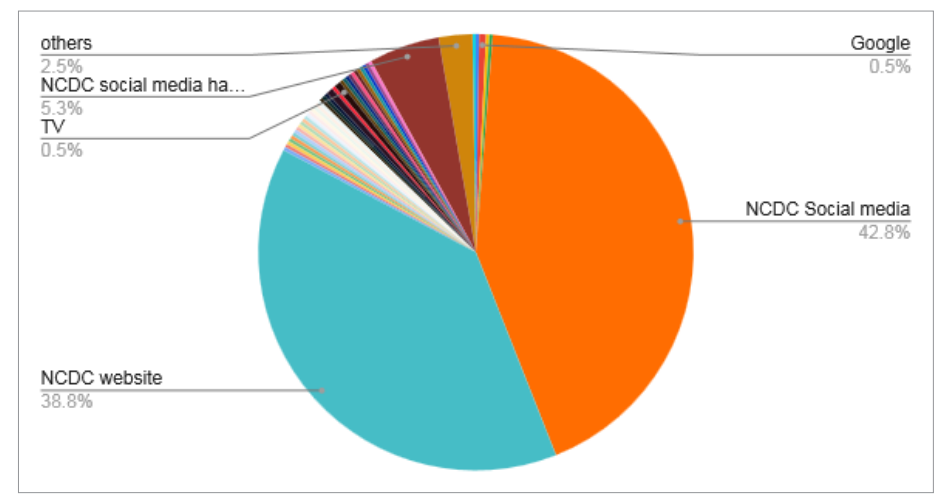

Figure 3. Where citizens go for NCDC COVID-19 information

Source: Fieldwork, 2020

Regarding the issue of information source, responses revealed platform options and awareness of NCDC communication during the COVID-19 lockdown. Interestingly, when looking for NCDC information on the Coronavirus, a higher percentage $(48.1 \%)$ of the sample population resorted to the NCDCs social media instead of the NCDC website, $38.8 \%$ of the population stated that they rely on the NCDC 
website when looking for information on Coronavirus. This is because deciding where people went to look for information on the COVID-19 during the lockdown will broadly reveal the public's platforms when looking for information on the Coronavirus. This will inadvertently prove or disprove public awareness of NCDCs COVID-19 messages during the lockdown.

This could mean that more people's need for interaction heightened during the lockdown amidst the uncertainties surrounding the Coronavirus and the social/ mobile restrictions. They preferred to look for NCDC's information on the Coronavirus via social media rather than radio, TV, or NCDC websites. This could have accounted for the higher social media users searching for NCDC information on the Coronavirus during the lockdown instead of the NCDC website when looking for NCDC's information on the Coronavirus. Besides the NCDC's social media and the NCDC website, other platforms noted by the respondents include; Google $(0.5 \%)$, TV $(0.5 \%)$, WhatsApp text messages, Nairaland, opera news, telegram, YouTube, online blogs, world health organisation and others.

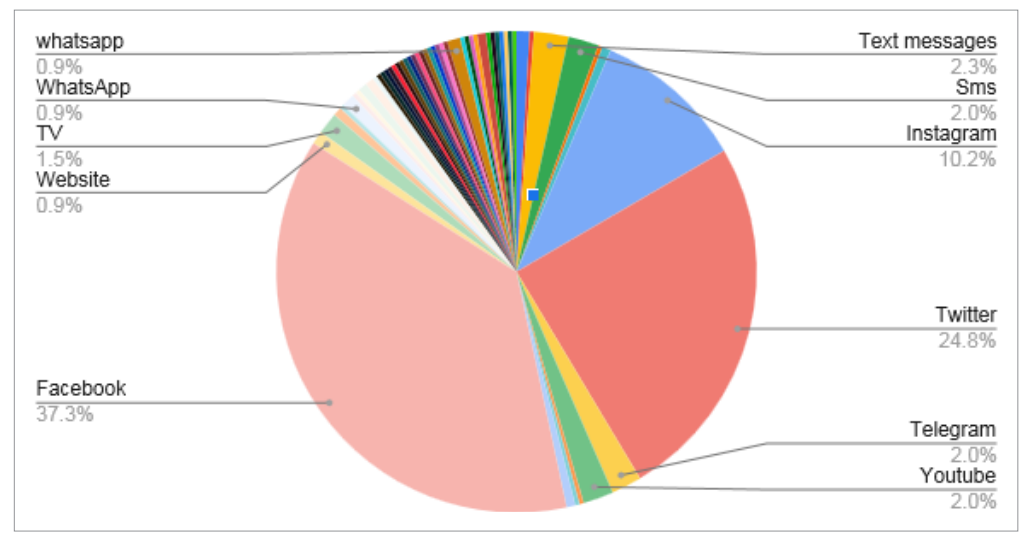

Figure 4. Social media platform NCDC COVID-19 messages was most seen

Source: Fieldwork, 2020

This inquiry was asked to narrow down public awareness of NCDC social media use (for COVID-19 communications during the lockdown) to the specific social media handles. Determining the social media platform with the highest public exposure of NCDC COVID-19 messages will help establish public awareness of NCDC communication during the lockdown and provide insight into which social media platform the public accessed COVID-19 information most. This is necessary to examine because it does not mean that the NCDC social media platform with the most significant following necessarily has the highest public awareness or public awareness of its messages. However, it is remarkable to note that the figures above correlate with the public followings of NCDC social media platforms. 
For instance, during the COVID-19 lockdown, most respondents saw NCDC COVID-19 messages on Facebook, Twitter, and Instagram. This is reflected as 37.3\% of the sample population said they saw NCDC messages the most on Facebook, $24.8 \%$ said they saw NCDC messages the most on Twitter, and $10.2 \%$ said they saw NCDC messages the most on Instagram. The rest of the respondents wrote that they saw NCDC messages the most via text, WhatsApp, TV, telegram, and YouTube. These figures align with existing reports on social media usage in Nigeria, where Facebook, Twitter and Instagram are among the top social media platform with the largest subscribers in Nigeria. Thus, determining the social media platform where NCDC COVID-19 messages were most seen will invariably measure NCDC social media and message awareness. The public can be aware of NCDC's social media platform and not aware of the message.

\section{Public Engagement with NCDC's Crisis Communication messages via social media during the lockdown}

Figure 5 shows the level of awareness of NCDC Facebook messages on COVID-19 during the lockdown. This was necessary based on preliminary research data showing that the NCDC social media platform with the most significant following is Facebook, with more than two million followers.

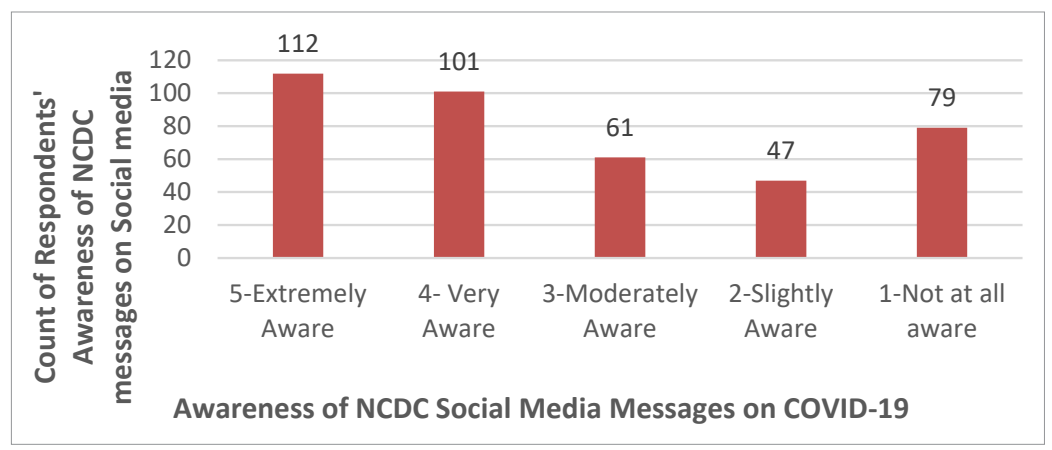

Figure 5. Awareness of NCDC Facebook messages on COVID-19 during the lockdown.

Consequently, in examining NCDC social media use, it was necessary to test awareness of messages disseminated via NCDCs social media platform (Facebook) with the largest following. Hence, the figures above show that during the lockdown, the largest number (28\%) responded that they were highly aware of NCDC Facebook messages on COVID-19, followed by those that were moderately aware 925.25\%). From the figures, those aware of NCDC Facebook messages on COVID-19 were more than those unaware. This could be attributed to a large number of Nigerians on Facebook. Based on Dataportal (2020), more than $80 \%$ of Nigeria social media users are Facebook. This proves a higher tendency for the Nigerians (active 
on social media) to see NCDC COVID-19 messages on Facebook. This can also explain the why NCDC Facebook platform has the most significant following beside the other social media platforms used by NCDC.

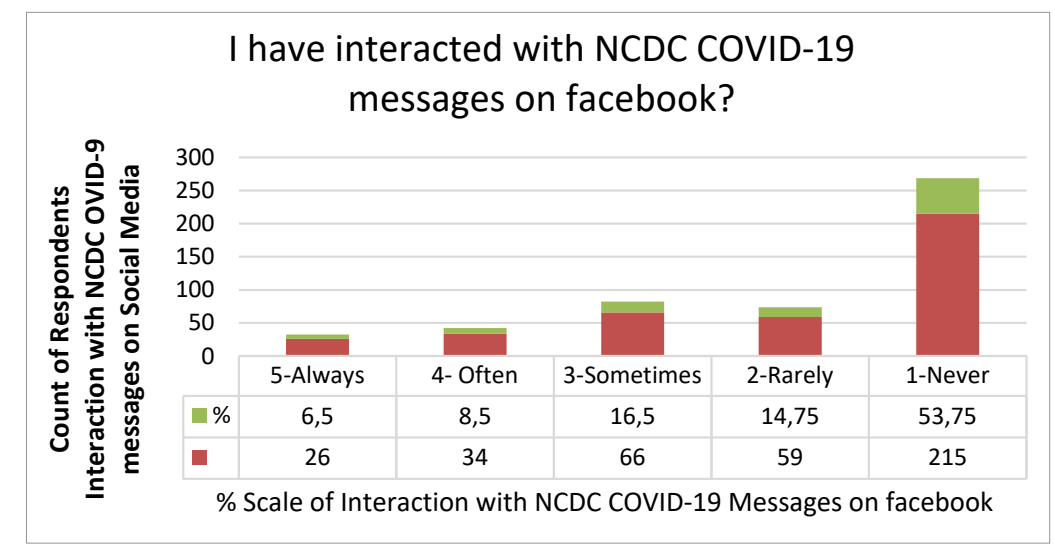

Figure 6. Public interaction with NCDC COVID-19 messages on Facebook

This question sought to determine the level of public interaction with NCDC COVID-19 messages on Facebook during the lockdown. Beyond seeing NCDCs Facebook messages on COVID-19, it is necessary to determine how effective these messages are based on public interaction. Based on the respondents' feedback, it is interesting that many of the respondents $(53.75 \%)$, which is more than half of the sample population, stated that they never interacted with NCDC COVID-19 messages on Facebook during the lockdown. While $14.75 \%$ stated they rarely interacted with NCDC COVID-19 messages on Facebook during the lockdown, 16.5\% said they sometimes interacted with NCDC COVID-19 messages. In addition, $8.5 \%$ said they often interacted with NCDC COVID-19 messages on Facebook during the lockdown. The least number of the respondents (6.5\%) said they constantly interacted with NCDC COVID-19 messages on Facebook during the lockdown.

Many people did not interact with NCDCs social media (Facebook) messages during the lockdown. Therefore, if most respondents did not interact with NCDC COVID-19 messages on Facebook during the lockdown, NCDCs messages are not interactive.

The above question was asked to determine public exposure to the different types of NCDC social media (Facebook) information on COVID-19. Based on preliminary research, most of the NCDC COVID-19 messages centred on; providing updates on the number of infected persons, knowledge on the mode of transmission, and preventive behaviours. Remarkably, more than half of the respondents $(50.2 \%)$ had seen NCDC information on the number of infected persons on social media-this could due to the daily NCDC updates/data on the number of infected persons. This is followed by $28.7 \%$ who had seen NCDCs information on preventive 
11. Which of the NCDC message types did I see on facebook during the lockdown?

400 responses

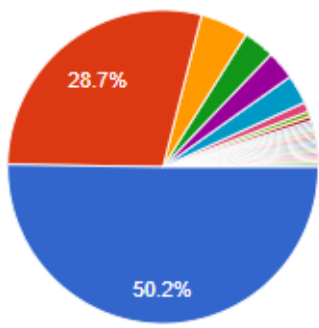

A) Information on number of infected...

B) Information on preventive behaviours

C) Information on mode of transmission

D) Others

All of the above

All

None

All of the above

A $1 / 4 \boldsymbol{\nabla}$

Figure 7. NCDC message types seen on Facebook during the lockdown

behaviours on social media. At the same time, very few persons (5.0\%) of the respondents had seen NCDC information on the mode of transmission. Also, very few respondents $(9.9 \%)$ had seen all, none or any other information on the different types of the NCDC COVID-19 during the lockdown. More of the respondents saw NCDC information on the number of infected persons on social media. However, very few of the respondents saw information on the mode of spread reflect NCDC COVID-19 communication effectiveness.

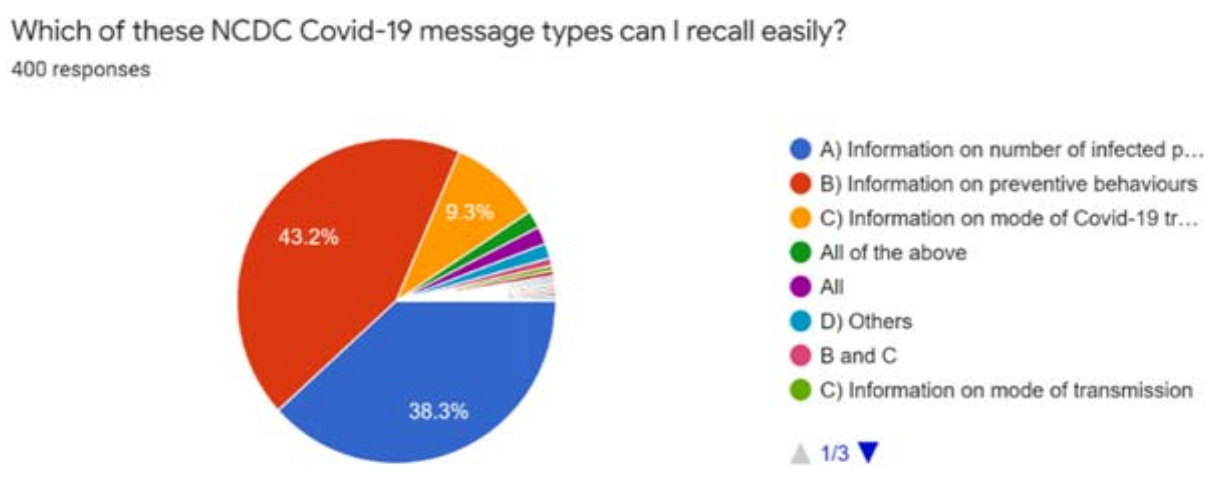

Figure 8. NCDC COVID-19 information on Facebook most easily recalled

Figure 8 shows which of the NCDC COVID-19 information on Facebook the public could easily recall. This question is a follow up from awareness of NCDC COVID-19 messages to recall. In contrast to responses in figure 7 , the figure above shows that more respondents $(43.2 \%)$ could easily recall NCDC information on preventive measures than those $(38.3 \%)$ who recall information on the number of infected persons. On the other hand, a very insignificant number of the respondents (9.139\%) could recall NCDC COVID-19 information on transmission mode. Also, a very few respondents $(9.1 \%)$ could recall all, none or any other information on the 
different types of the NCDC COVID-19 during the lockdown. This indicates that more respondents recall NCDC Facebook information on COVID-19 preventive measures than information on the number of infected persons. Thus, the findings in figure 8 contrast with findings in the previous figure 7 . This limits the engagement of NCDC social media information on COVID-19 during the lockdown.

\section{Evidence determining if the NCDC social media use for COVID-19 crisis} communication influenced positive health behaviour during the lockdown

Figure 9 bellow measures how much the NCDC social media messages on COVID-19 affected public opinion about the Coronavirus. At the coronavirus outbreak in Nigeria, there were different opinions about the Coronavirus on social media. However, these opinions were based on speculations, social media conversations, and unreliable information about the Coronavirus.

In Nigeria, the NCDC is the central government parastatal recognised as the goto place for information and enlightenment on the Coronavirus. Therefore, amidst all the unreliable social media information on COVID-19, it is vital to determine to what extent the NCDC social media messages on COVID-19 affect public opinion about the Coronavirus.

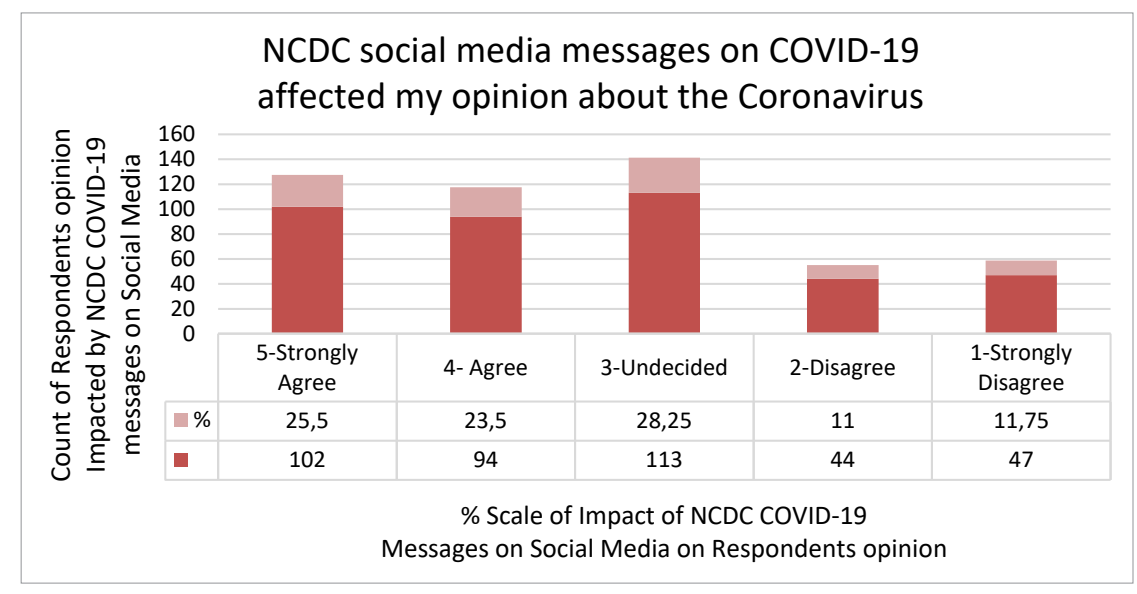

Figure 9. Effect of NCDC social media messages on COVID-19 public opinion about the Coronavirus

Based on the figure above, $28.5 \%$ of the respondents are undecided about whether the NCDC social media messages on COVID-19 affected their public opinion about the Coronavirus. In comparison, $25.5 \%$ strongly agree, $23.5 \%$ agree, and $11.75 \%$ strongly disagree, and $11 \%$ agree. The figures indicate that not much public opinion about the Coronavirus has been affected by the NCDC social media messages on COVID-19. The highest number of respondents claim that they are still undecided on the effect of NCDC social media messages on their opinion about the Coronavirus. 


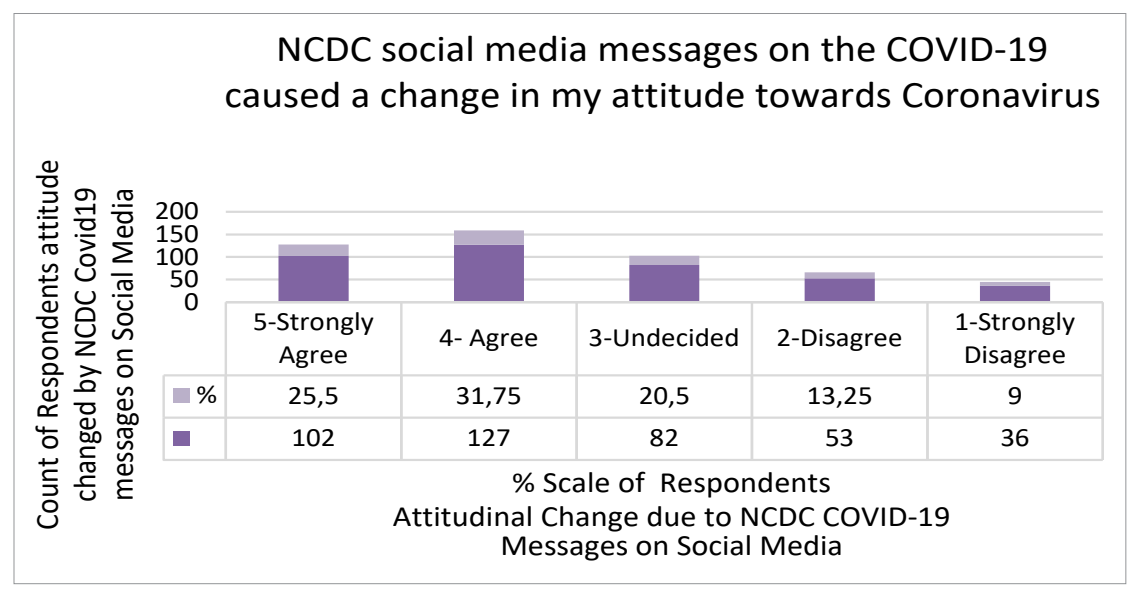

Figure 10. Effect of NCDC social media messages on the COVID-19 on public attitude

Source: Fieldwork, 2020

Beyond determining how much the NCDC social media messages on COVID-19 affected public opinion about the Coronavirus, it was necessary to narrow the examination to determine if NCDC social media messages on the COVID-19 influenced a change in public attitude towards the Coronavirus. Based on the figure above, more than half of the respondents, $57.25 \%$, agree and strongly agree that NCDC social media messages on the COVID-19 caused a change in their attitude towards the Coronavirus. While 42.75 are still undecided, they disagree strongly that they have changed their attitude due to NCDC social media messages on COVID-19, which means the gap between those who have experienced an attitudinal change due to NCDC social media messages on COVID-19 is not much.

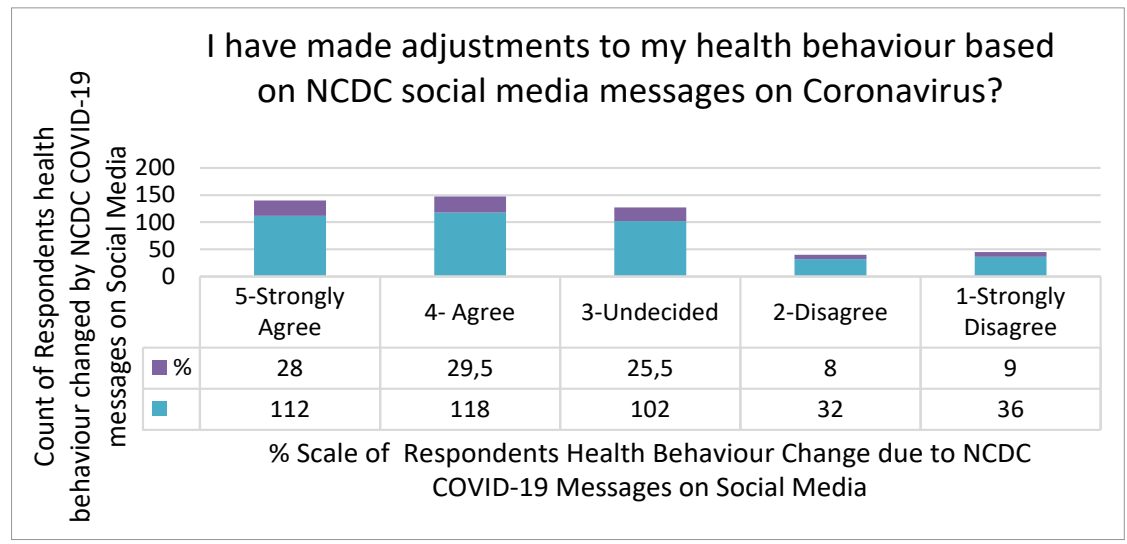

Figure 11. Public health adjustments based on NCDC social media messages on Coronavirus 
To further determine that any change in attitude towards the Coronavirus is connected to public response via adjustments in health behaviour, it was necessary to determine if the public had adjusted health behavior based on NCDC social media messages Coronavirus. The figure above shows that a quarter of the respondents $(25.5 \%)$ are undecided about any adjustments in their health behaviour due to NCDC social media messages on Coronavirus. In comparison, $28 \%$ of the respondents strongly agree that they had adjusted health behaviour based on NCDC social media messages on Coronavirus. These figures show that there is not much gap between undecided and those who strongly agree that they had improved health behaviour based on NCDC social media messages on Coronavirus.

\section{Summary of NCDC messaging impact on the public health behaviour}

The following figure (Figure 12) is a descriptive summary of the impact of NCDC crisis communication messaging on general public health behaviour. This study focused on examining the public response to NCDC COVID19 messages on public health.

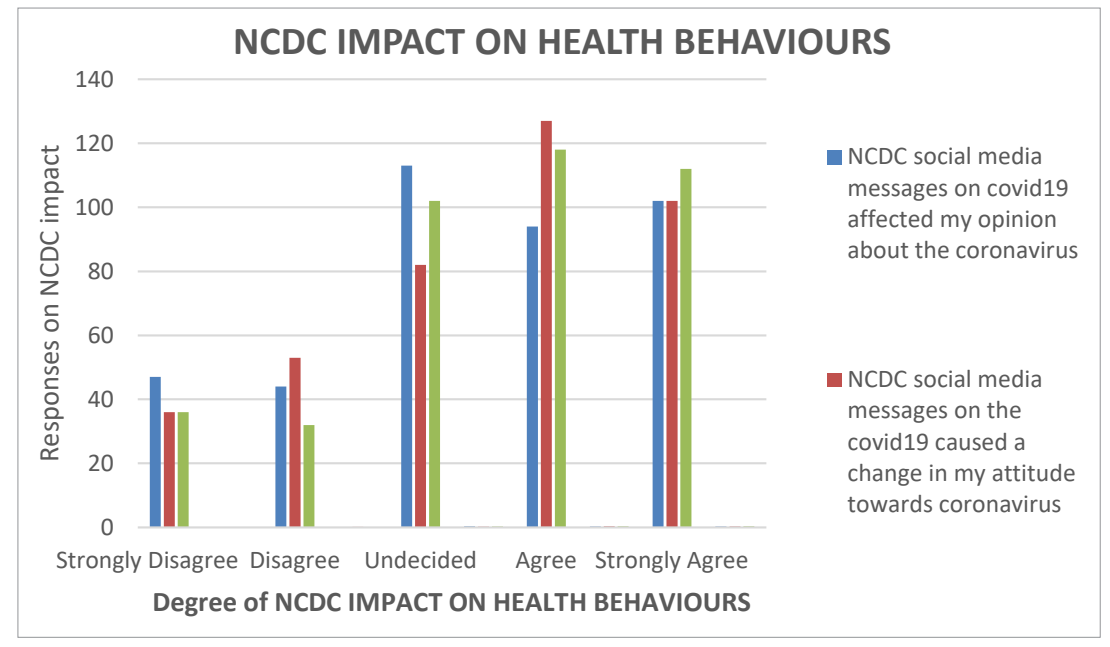

Figure 12. NCDC Communication Impact on Health Behaviours

Source: Fieldwork, 2020

Notably, a more significant part of the respondents is undecided on whether the NCDC social media messages on COVID-19 affected their opinion. In comparison, a more significant part of the respondents agrees that the NCDC social media messages on COVID-19 affected their attitude. Also, a significant number of the respondents also agree that they have adjusted their health behaviours in response to NCDC social media messages on COVID-19. 


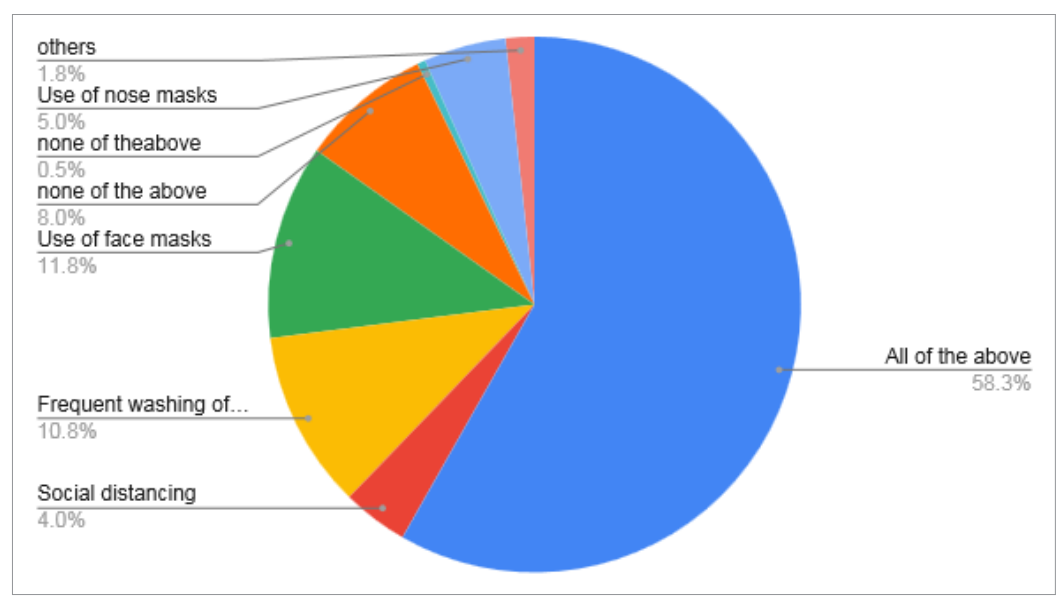

Figure 13. Positive public health adjustment due to NCDC social media communications on COVID-19

Figure 13 show findings of a follow-up question aimed to determine specific health behavioral adjustments made by the public in response to NCDC social media messages on COVID-19. A majority of the respondents (58.3\%) responded to social media messages on COVID-19 by adhering to the three primary health preventive measures against COVID-19, which includes; frequent handwashing with water or use of alcohol-based sanitiser, observing physical distancing, use of face mask in public places. This final question sought to know the public view of NCDC COVID-19 messages via social media. Again, the respondent views differed as some believe that the NCDC COVID-19 messages via social media are consistently informative, apt and enlightening. While others believe it is an avenue for the government to siphon funds and believe that the NCDC could do more.

\section{Discussion}

As a follow-up on the findings above, it is noteworthy that social desirability may have come to play in the attention of respondents that they developed positive health behaviours. Some respondents tend to report an answer in a way that they believe is more socially acceptable than their "actual" answer due to social desirability. They do this in order to portray a positive image of themselves and avoid unfavourable feedback. This outcome is probable in this study because positive health adjustments has been recommended by the World Health Organisation, other health bodies and governments (even with punitive measures to ensure compliance). Although social desirability presents a form of bias, the advantage of this study is that positive health behaviour has being reinforced in the sample population and this is a plus. The next few paragraphs discuss findings as per the research questions of the study. 
Research question 1: What is the level of public awareness on NCDC COVID-19 crisis communication via Facebook during the COVID-19 lockdown?

This research found out that there is a high level of public awareness of NCDC social media communications during the Coronavirus lockdown. This is because, out of most respondents who said they are aware of the NCDC, more than one third (42.8\%) indicated that they went to NCDC social media handles to get COVID-19 during the lockdown. This is interesting because it will be expected that many people will prefer to look for NCDC COVID-19 communication via the NCDC website.

However, many respondents were more aware of NCDC COVID-19 communications on social media than on conventional or other media. This may be due to the need for public engagements that social media provides, especially during a lockdown period. This aligns with the study by Schultz \& Wehmeier (2010), where they affirm that social media have a predominant role in crisis communication as they help create virtual communities. Based on this, public awareness of NCDC COVID-19 communication can be easily connected to the significant role of building virtual communities, which the social media platforms provide.

This study's time frame was during the first phase of the COVID-19 lockdown period in Nigeria, characterised by social restrictions. Therefore, it would make sense that a large chunk of the public would be aware of NCDC social media communications on the COVID-19 as they were going to the social media handles to look for communications on COVID-19 during the lockdown. This ties up with the findings of Apuke \& Tunca (2018), which states that social media effectively connects an organisation and its public when a crisis occurs. This captures social media as a more manageable platform to create communities. Thereby creating a higher need for virtual communities and social interaction and making communication on this platform more visible to the public.

Furthermore, Civelek, Cemberci, \& Eralp (2016) support this view in their studies on social media role in crisis communication by pointing out that social media's striking features. They include; interactivity, capacity for messages to travel long distances, shareability and collective consciousness. These make it easy for communication to spread quickly, thus increasing public awareness of communication passed via these platforms. These features inherent in social media make it easy for the public to be aware of NCDC COVID-19 communications.

Unlike the mainstream media, which lacks interactivity, shareability or virtual community platform. As reflected in this study, many of the respondents were unaware of NCDC communications via social media but equally confirmed specific social media platforms they saw NCDC messages the most. As seen from the analysis, $37.3 \%$ of the sample population said they saw NCDC messages the most on Facebook, $24.8 \%$ said they saw NCDC messages the most on Twitter, and $10.2 \%$ said they saw NCDC messages the most on Instagram. These confirm that the public is aware of NCDC COVID-19 communications during the lockdown. 
Research question 2: What is the level of engagement between NCDC COVID-19 crisis communication via Facebook and Nigerians?

It was necessary to test NCDC communications engagement on COVID-19 via a specific social media platform-(Facebook). Based on the analysis, it is interesting to discover that even though many people are aware of NCDC COVID-19 messages on Facebook, as seen in figure 9; however, it is noticeable that a large number of the respondents (53.75\%) did not interact with NCDCs Facebook messages during the lockdown. This finding shows that the NCDC communications on COVID-19 via social media (Facebook) are not highly engaging. Because engagement largely determined by measured in use. Therefore, if many people are aware of NCDC Facebook messages on COVID-19 but do not interact with such messages, then the engagement level of such messages is low. Preliminary research data on social media usage in Nigeria shows that Facebook has the largest number of users in Nigeria. Based on Dataportal (2020) report, more than $80 \%$ of Nigerians on social media are Facebook users. Also, preliminary observations by the researcher show that NCDC social media platform with the most significant following is Facebook, with more than two million followers (Nigeria Centre for Disease Control-Home, Facebook, n.d.). These background studies show the opportunities inherent in this social media platform for effective social media use. Therefore, finding that people do not interact with NCDC messages questions NCDC's engagement as it opposes Watkins (2014) study that point out that social media are most often interactive and dialogic. This is further confirmed by the responses to questions that dealt with NCDC message types seen and recalled by the public. This was equally used to test the public engagement of NCDC communications on COVID-19 on Facebook.

Based on preliminary research, most of the NCDC COVID-19 messages centred on; providing updates on the number of infected persons, knowledge on the mode of transmission, and preventive behaviours. While most respondents saw information on the number of infected persons, more people could recall preventive behaviours. The findings here connect with the conclusions in the figures above, showing that many people could see updates on the number of infected persons. Still, it was only informational and not interactive.

This interactivity limitation is perhaps attributable to the effects of plenty of sources of information on COVID-19 via social media. Studies term this situation as an 'infodemic'. The search for COVID-19 updates on the internet jumped from 50\%-70\% across all generations (Pan American Health Organisation, 2020). This misinformation in a pandemic leads to many false or misleading stories on the Coronavirus being fabricated and shared on social media without any background or quality check. With this background information on the avalanche of social media communications on COVID-19, it was evident that NCDC simply generating data updates on COVID-19 and creating awareness is not engaging enough. They were unable to create public interaction and usage of the information and enlightenment provided. 
This finding also reflects Coombs \& Holladay (2010) views on one of the two main types of crisis communication known as crisis knowledge which focuses on collecting information, sharing knowledge, analysing information (knowledge creation). This is proven as many respondents could recall information on preventive behaviours, which was more practical and interactive than the daily updates. Thus, the public engagement level of NCDC social media communication on COVID-19 is low.

Research question 3: What is the influence of NCDC crisis communication via

Facebook on Nigerians' (reported) positive health behavior?

Findings from the study show that social media use for NCDC social media communication influenced positive health behaviour significantly. Even though large parts of the respondents are undecided on whether the NCDC social media messages on COVID-19 affected their opinion. However, a more significant portion of the respondents agrees that the NCDC social media communication on COVID-19 affected their attitude. Furthermore, a more significant number of the respondents also agree that they adjusted their health behaviour in response to NCDC social media messages on COVID-19.

Although, there has been public attitudinal change and behavioural adjustments. However, many respondents are still uncertain about change in public opinion about the Coronavirus due to NCDC communications on social media. The ability of the NCDC communications on social media to affect public opinion about the Coronavirus means an ability to frame public opinion. This is supported by Coombs \& Holladay (1996). They rightly pointed out that the aim of communication during a crisis is to shape how the public view or understand crisis and how the public also perceives an organisation during a crisis.

Furthermore, based on the health belief theory that links the likelihood of an individual to engage in preventive health behaviour to perceived susceptibility, severity, benefits and barriers, it is evident that the attitudes and behaviour of the public towards the Coronavirus is primarily influenced by the NCDC information accessible to them. From the finding, the NCDC information available through social media greatly influenced public response and behaviour.

\section{Conclusion and recommendations}

This study explored NCDC social media use for crisis communication during the Coronavirus outbreak in Nigeria by determining the level of awareness, engagement and positive health behaviour change based on NCDC social media communications in Nigerians. The research aimed to discover three things: public awareness of NCDC social media communications during the Coronavirus lockdown, the engagement level of NCDC Facebook communication on COVID-19 and how much the NCDC social media for communication has influenced positive health behaviour. 
The study reviewed relevant literature, which showed that the Coronavirus outbreak turned into a pandemic that affected nations, including Nigeria. This was evident in the drastic changes in the country's economic, social, and industrial activities. Amongst which are heightened uncertainties about the spread of the Coronavirus and restriction of movements caused by the lockdown measures. Such curfews restricted movements limited social interactions and affected Nigeria's economy as many private organizations laid-off workers.

Social media's strategic role in the public domain cannot be overemphasized as it becomes a powerful tool of information and communication during a crisis. However, in managing this situation, communication is inevitable as it is vital to shaping its perception. This involves creating a dominant narrative about what happened, updates, what should be done, and what can be done to manage the situation in achieving this. It also directly affects how crisis information is spread and how it influences the general public. Hence, providing the right and timely information through effective communication and ensuring that the information collected is processed into knowledge and shared with others to change behaviour are some of the issues reviewed via existing literature.

However, this study focused on social media use for communications during the COVID-19 lockdown via the NCDC. Therefore, it was necessary to determine if the NCDC social media use for crisis communication during the Coronavirus outbreak in Nigeria was effective, had public awareness and caused positive public behavioural adjustments. To determine this, a sample population of the study consisted of active people on social media. The methods used for the study is the survey methods, using an online questionnaire.

The study's findings generally agreed with existing literature regarding the relevance of social media use for crisis communication. It discovered that social media interactive features become potent for public awareness and behavioural adjustments during crises. One significant finding of this study was that social media use during crises is more effective when it induces public engagement via practical communication and data-driven or informational communication. This research showed a disparity between the significant people who saw NCDC communications on COVID-19 updates but could not recall it. However, they could remember NCDC communications on COVID-19 preventive measures because the communication involved practical activities. The many undecided respondents about a change in opinion may be attributed to the avalanche of interactive COVID-19 communications competing for public buy-in on social media.

During a public health crisis such as a disease outbreak or pandemic, social media use is an understudied field in academics. As nations continue to devise ways to manage the initial tension, anxiety and uncertainties surrounding the Coronavirus outbreak, effective communication remains an essential tool. However, how this tool is deployed will largely determine public awareness, information, and enlightenment 
about the Coronavirus and inadvertently influence general public behaviour and adjustments in managing the COVID-19 pandemic.

This study's findings show that the public knows about the NCDC. There was also a high level of public awareness of the NCDC social media use for public communications during the Coronavirus outbreak. However, beyond this awareness of NCDC social media communication, its engagement is questionable as there is a lack of interactive communication.

Social media is a potent tool for communication during a public health crisis. It can influence public attitudes and induce behavioural adjustments required for the management of a disease spread. For instance, more public consciousness and adoption of disease preventive behaviour, such as face mask, social distancing, and frequent washing of hands. In the case of the Coronavirus pandemic in Nigeria, this study discovered that there had been a positive adjustment in public behaviour based on NCDC social media communications on Coronavirus.

Myriads of social media opinions on an issue can compete strongly with reliable information and cause public uncertainty about genuine, fact or false information. For example, during the lockdown, there was an intensity of public communications on the Coronavirus; however, NCDC social media messages (focusing on Facebook) were not interactive, resulting in uncertainty about COVID-19 information NCDC.

The study concedes to certain limitations. These include those inferences on the behavioral attitude of the population towards COVID-19 updates by NCDC are solely based on a survey of the sample population across the country. Further study can incorporate input or primary data from the NCDC office.

Also, it is worth noting that the identified changes are self-reported behaviour. Furthermore, it is probable that social desirability was at play in discussing opinions and impact of NCDC crisis communication. However, the weight of findings trumps these limitations.

Based on the findings of the research, recommendations are as follows. During public health crises (such as during the Coronavirus outbreak), there is usually an influx of information. Amid the competing information and intense social media conversations, there is a need to ensure that public communications go beyond generating awareness to engaging the public. Public engagement suggests positioning messages that encourage public interaction. Therefore, the NCDC communications and such agencies internationally should inform or enlighten the public and encourage public use and exchange of such messages.

Relying on some public observations, there are suggestions for the NCDC to do more to re-sensitise the society, reduce information monotony-meaning to diversify information/communication style, increase communications on preventive measures rather than the number of infected persons and death

Finally, there may be a need for future studies; to investigate why NCDC COVID-19 messages did not significantly affect Nigerians' opinion about the Coronavirus. Is 
this a question of public distrust or an avalanche of information on social media? Another research angle is an investigation into the impact of infodemics on the spread of COVID-19.

\section{Compliance with Ethical Standards}

The authors declare that there is no conflict of interest as regards this study. It is a self-funded and independent study. The inquiry into NCDC's communication activities does not breach any intellectual property right or copyrights. Also, respondents filled the survey free of charge and without pressure or anticipation of compensation.

\section{References}

1. Apuke, O. D., \& Tunca, D. E. (2018). Social Media and Crisis Management: A Review And Analysis Of Existing Studies. Laü Sosyal Bilimler Dergisi, IX-II, 199-115.

2. Banerjee, D., \& Meena, K. S. (2021, March 18). COVID-19 as an "Infodemic" in Public Health: Critical Role of the Social Media. Frontiers in Public Health, 9, 610-623. https:// doi.org/10.3389/fpubh.2021.610623

3. BBC-British Broadcasting Corporation. (2020, March 21). Coronavirus: Italian wey first test positive for COVID-19 inside Nigeria don discharge from Lagos Isolation ward. Retrieved June 28, 2021 from https://www.bbc.com/pidgin/tori-51986476

4. Bubeck, P., Wouter Botzen, W. J., Laudan, J., Aerts, J. C. J. H., \& Thieken, A. H. (2018). Insights into Flood-Coping Appraisals of Protection Motivation Theory: Empirical Evidence from Germany and France. Risk Analysis, 38(6), 1239-1257. https://doi.org/10.1111/ risa. 12938

5. Chen, K., Luo, Y., Hu, A., Zhao, J., \& Zhang, L. (2021, May 10). Characteristics of Misinformation Spreading on Social Media During the COVID-19 Outbreak in China: A Descriptive Analysis. Risk Management and Healthcare Policy, 14, 1869-1879. https://doi. org/10.2147/RMHP.S312327

6. Civelek, M. E., Cemberci, M., \& Eralp, N. E. (2016). The Role of Social Media in Crisis Communication and Crisis Management. International Journal of Research in Business \& Social Science, 5(3), 111-120.

7. Coombs, W. T., \& Holladay, S. J. (1996). Communication and Attributions in a Crisis: An Experimental Study in Crisis Communication. Journal of Public Relations Research, 8(4), 279-295.

8. Coombs, W. T., \& Holladay, S. J. (2010). The Handbook of Crisis Communication. United Kingdom: Wiley-Blackwell.

9. Datareportal. (2020). Digital 2020 Nigeria. Datareportal.com. Retrieved from https://data reportal.com/reports/digital-2020-nigeria\#

10. Dunbar, R., Arnaboldi, V., Conti, M., \& Passarella, A. (2015). The Structure of Online Social Networks Mirrors Those in the Offline World. Social Networks, 43(5), 39-47. 
11. Ghebreyesus, T. A. (2020). WHO Director-General's opening remarks at the media briefing on COVID-19-11 March 2020. WHO Director General's Speeches. Retreived from https://www.who.int/director-general/speeches/who-director-general-s-openingremarks-at-the-media-briefing-on-covid-19---11-march-2020\%0Ahttps:/www.who.int/ director-general/speeches/detail/who-director-general-s-opening-remarks-at-the-me dia-briefing-on-c

12. Janmaimool, P. (2017). Application of Protection Motivation Theory to Investigate Sustainable Waste Management Behaviours. MPDI, 2-16.

13. NCDC. (2020, 4 September). COVID-19 NIGERIA. Retrieved from https://COVID-19.ncdc. gov.ng/

14. Nigeria Centre for Disease Control - Home | Facebook. (n.d.). Retrieved July 2, 2021, from https://www.facebook.com/NCDCgov

15. Pan American Health Organisation. (2020). Understanding the Infodemic and Misinformation in the Fight against COVID-19. Retrieved from https://iris.paho.org/bitstream/ handle/10665.2/52052/Factsheet-infodemic_eng.pdf?sequence=14\#: :text=infodemic\% $3 \mathrm{~A} \% 20$ an\%20overabundance $\% 20$ of\%20information,increase $\% 20 \mathrm{in} \% 20$ the $\% 20$ volume $\% 20$ of: PAHO \& WHO.

16. Rogers, R. W. (1975). A Protection Motivation Theory of Fear Appeals and Attitude Change1. The Journal of Psychology, 91(1), 93-114. https://doi.org/10.1080/00223980.19 75.9915803

17. Rogers, R. W., \& Prentice-Dunn, S. (1997). Protection motivation theory. In D. S. Gochman (Ed.), Handbook of health behavior research 1: Personal and social determinants (pp. 113132). Plenum Press.

18. Sahni, H., \& Sharma, H. (2020). Role of social media during the COVID-19 pandemic: Beneficial, destructive, or reconstructive? International fournal of Academic Medicine, 6(2), 70-75.

19. Shahaduzzaman, D., Rahman, S., \& Mehnaz Rabbani. (2020, 2 May). Crisis of Communication during COVID-19: A Rapid Research. BRAC Institute of Government and Development. Retrieved from https://bigd.bracu.ac.bd/study/crisis-of-communication-duringCOVID-19-a-rapid-research/

20. Shaw, A. (2012, 31 December). Protection Motivation Theory. Strategic Planet. Retrieved from https://www.strategic-planet.com/2012/12/protection-motivation-theory/

21. Shereen, A. M., Suliman, K., Abeer, K., Nadia, B., \& Rabeea, S. (2020). COVID-19 infection: Origin, transmission, and characteristics of human Coronaviruses. Fournal of Advanced Research, 24, 91-98.

22. Schultz, F., \& Wehmeier, S. (2010). Online Relationships. Manual Online Conversation, 6(1), 409-433.

23. Tran, T., Valecha, R., \& Rad, P. \&. (2020). An Investigation of Misinformation Harms Related to Social Media during Two Humanitarian Crises. Information Systems Frontiers. https://doi.org/10.1007/s10796-020-10088-3

24. Watkins, B. (2014). An Integrated Approach to Sports Branding: Examining the Influence of Social Media on Brand Outcomes. International fournal of Integrated Marketing Communications, 6(2), 18-25. 
25. WHO (World Health Organisation). (2020, 3 September). Coronavirus disease (COVID-19) pandemic. Retrieved 3 September 2020, from https:/www.who.int/health-topics/ Coronavirus\#tab=tab_1

26. WHO (World Health Organisation). (2020, 23 April). Coronavirus disease 2019 (COVID-19) Situation Report - 94. Retrieved from https://www.who.int/docs/default-source/ Coronaviruse/situation-reports/20200423-sitrep-94-COVID-19.pdf

27. WHO (World Health Organisation). (2020, March). WHO director-general's opening remarks at the media briefing on COVID-19-11 March 2020. WHO | World Health Organization. Retrieved from https://www.who.int/director-general/speeches/detail/who-director-general-s-opening-remarks-at-the-media-briefing-on-covid-19---11-march-2020

28. Wong, T. S., Gaston, A., DeJesus, S., \& Prapavessis, H. (2016). The utility of a protection motivation theory framework for understanding sedentary behavior. Health Psychology and Behavioral Medicine, 4(1), 29-48. https://doi.org/10.1080/21642850.2015.1128333

29. Zimmer-Gembeck, M. J., \& Skinner, E. A. (2016). The Development of Coping: Implications for Psychopathology and Resilience. In Developmental Psychopathology (pp. 1-61). John Wiley \& Sons. https://doi.org/10.1002/9781119125556.devpsy410 